\title{
AFP role in predicting recurrence of hepatocellular carcinoma after living donor liver transplantation in HCV patients
}

\author{
A. L. KOMOROWSKI ${ }^{1}$, C.-C. HSU², K. D. JULKA³, B. VASAVADA ${ }^{4}$, C.-C. LIN $^{5}$, C.-C. WANG ${ }^{5}$, C.-L. CHEN ${ }^{5}$
}

${ }^{1}$ Department of Surgical Oncology, Maria Skłodowska-Curie Memorial Cancer Centre, Kraków, Poland; ${ }^{2}$ Department of Nuclear Medicine, Kaohsiung Chang Gung Memorial Hospital, Kaohsiung, Taiwan; ${ }^{3}$ Department of Surgery, Salmaniya Medical Complex, Bahrain; ${ }^{4} H P B$ surgery and Liver transplantation, Continental Hospitals, Hyderabad, India; ${ }^{5}$ Liver Transplantation Program and Department of Surgery, Kaohsiung Chang Gung Memorial Hospital, Chang Gung University College of Medicine, Kaohsiung, Taiwan

*Correspondence: alkomorowski@wp.pl

Received March 15, 2017 / Accepted August 16, 2017

\begin{abstract}
HCC is one of the leading causes of death worldwide. Liver transplantation including living donor transplantation is the best available treatment. We have analyzed our experience with LDLT (living donor liver transplantation) in patients with $\mathrm{HCC}$ and $\mathrm{HCV}$ in order to determine if alpha feto-protein (AFP) is a better predictor of recurrence than the tumor burden. We have identified all patients with HCV related liver disease and HCC who have undergone LDLT in one center during the period from December 2000 to December 2014. Outcomes from the prospective database were compared for patients who met Milan criteria (single tumor $\leq 5 \mathrm{~cm}$, maximum of 3 total tumors with none $>3 \mathrm{~cm}$ ) or not. Uni- and multi-variable analyses of factors influencing recurrence free survival (RFS) were performed. A total of 142 patients with HCC and HCV associated liver disease underwent LDTL during the study period. RFS was $96.4 \%$ at 1 year, $91.8 \%$ at 3 years and $91.8 \%$ at 5 years. Gender, Model for End-stage Liver Disease (MELD), pre-transplant therapy, AFP level, tumor number, total tumor size were predictors of recurrence in univariable analysis. In multivariable analysis MELD score (Hazard ratio (HR) 1.16) and $\log 10$ AFP (HR 3.14) were predictors of RFS. In the ROC curve analysis with an AUC of 0.76 the optimal cut-off value of AFP was $26 \mathrm{ng} / \mathrm{mL}$. In conclusion, MELD score and pre-transplant AFP predict recurrence after LDLT for HCC with HCV infection.
\end{abstract}

Key words: liver transplantation, HCC, AFP, liver cancer, recurrence

Hepatocellular carcinoma (HCC) is one of the leading causes of cancer death worldwide [1]. The possibility of liver resection is restricted in patients with cirrhotic livers due to poor future remnant liver function. Since the introduction of the Milan criteria [2], liver transplantation (LT) has become a valuable option for selected HCC patients. For those patients, LT is the best treatment possibility as it cures both HCC and the underlying liver disease. However, the shortage of deceased liver donors is a strong limiting factor to this approach. The living donor liver transplantation (LDLT) has been proposed as an option for HCC patients with Child B or C liver disease. Several preoperative factors have been suggested to identify patients that have the highest probability of cure with LDLT. Most commonly used systems are the Milan criteria [2] and University of California, San Francisco (UCSF) criteria [3]. Both systems base the identification of patients suitable for LDLT on a tumor burden. However, it has been recently suggested that preoperative alpha feto-protein (AFP) level can better predict recurrence and survival after LT for HCC than the tumor burden [4]. Patients with hepatitis $\mathrm{C}$ virus (HCV) infection are a group with generally poorer prognosis after LT than patients with no HCV infection [5]. In this paper, we try to establish the role of pre-transplant AFP level in the subpopulation of patients with HCC and HCV infection.

\section{Patients and methods}

Patient selection. From December 2000 to December 2014 we have performed 1153 LDLT. In the prospective database we have identified all patients who underwent LDLT for HCC and had an underlying HCV infection. The data on 142 patients was analyzed. Seventy-six percent of patients fulfilled UCSF criteria for transplantation (solitary tumor measuring up to $6.5 \mathrm{~cm}$ in diameter or up to three lesions, each measuring no more than $4.5 \mathrm{~cm}$ in diameter, with a total combined measurement of less than $8 \mathrm{~cm}$ ) while $35 \%$ did not meet the Milan criteria (single tumor $\leq 5 \mathrm{~cm}$, 
maximum of 3 total tumors with none $>3 \mathrm{~cm}$ ). The hepatitis $\mathrm{B}$ virus (HBV) infection coexisted in $16 \%$ of patients. All patients were operated by the same team using the standardized technique of LDLT described elsewhere [6]. All arterial and biliary anastomoses starting from 2006 have been performed by the same microsurgeon. Before this date it had been done in a standardized fashion by a liver surgeon using surgical magnifying loops [7].

The following variables were analyzed as potential factors influencing recurrence free survival (RFS): pre-transplant AFP level, age, sex, the Model for End-Stage Liver Disease (MELD) score, number of tumors, total tumor size, bridge therapy before transplantation, diabetes, Milan criteria, coexisting $\mathrm{HBV}$ infection. AFP levels obtained directly

Table 1. Patient demographics and pre-transplant clinical characteristics.

\begin{tabular}{|c|c|}
\hline Variables & Patients $(n=142)$ \\
\hline \multicolumn{2}{|l|}{ Age (years) } \\
\hline mean $(\mathrm{SD})$ & $57.5(5.9)$ \\
\hline range & $44.8-68.8$ \\
\hline \multicolumn{2}{|l|}{ Gender } \\
\hline Man & $93(65.5 \%)$ \\
\hline Woman & $49(34.5 \%)$ \\
\hline \multicolumn{2}{|l|}{ Diabetes } \\
\hline Yes & $36(25.4 \%)$ \\
\hline No & $106(74.6 \%)$ \\
\hline \multicolumn{2}{|l|}{ CTP score } \\
\hline A & $50(35.2 \%)$ \\
\hline B & $64(45.1 \%)$ \\
\hline $\mathrm{C}$ & $28(19.7 \%)$ \\
\hline \multicolumn{2}{|l|}{ MELD score } \\
\hline Median (IQR) & $11(5)$ \\
\hline Range & $6-37$ \\
\hline \multicolumn{2}{|c|}{ Combine HBV infection } \\
\hline Yes & $24(16.9 \%)$ \\
\hline No & $118(83.1 \%)$ \\
\hline \multicolumn{2}{|c|}{ Pre-transplant therapy } \\
\hline Yes & $105(73.9 \%)$ \\
\hline Hepatectomy & $22(15.5 \%)$ \\
\hline TAE & $71(50.0 \%)$ \\
\hline RFA & $12(8.5 \%)$ \\
\hline PEI & $62(43.7 \%)$ \\
\hline No & $37(26.1 \%)$ \\
\hline \multicolumn{2}{|c|}{ Pre-transplant AFP (ng/ml) } \\
\hline Median (IQR) & $14.2(37.0)$ \\
\hline Range & $3-37867$ \\
\hline$<20$ & $88(62.0 \%)$ \\
\hline $20-200$ & $44(31.0 \%)$ \\
\hline$>200$ & $10(7.0 \%)$ \\
\hline
\end{tabular}

SD, standard deviation; CTP, Child-Turcotte-Pugh score; MELD, Model for End-Stage Liver Disease; IQR, interquartile range; HBV, hepatitis B virus; TAE, transcatheter arterial embolization; RFA, radiofrequency ablation; PEI, percutaneous ethanol injection; AFP, serum $\alpha$-fetoprotein. before surgery were taken into account. For the MELD score, we used the score calculated before MELD except for HCC. Bridge therapies were: radiofrequency ablation (RFA), percutaneous ethanol injection (PEI), transarterial chemoembolisation (TACE), surgical resection.

Statistics. Categorical variables are expressed as percentages, and continuous values are expressed as means (standard deviation, SD) or median (interquartile range, IQR) if data were not normally distributed. Categorical variables were compared using $\chi^{2}$ test or Fisher's exact test as appropriate. Continuous variables were compared between groups using the $t$ test or Mann-Whitney $U$ test as appropriate. The KaplanMeier method was used to estimate overall survival (OS) and RFS probabilities. RFS time was defined as the interval between the date of LDLT and the date when recurrence was detected by computed tomography $(\mathrm{CT})$, magnetic resonance imaging (MRI), or ${ }^{18} \mathrm{~F}$-fluorodeoxyglucose positron emission tomography (FDG-PET). Patients' deaths unrelated to HCC recurrence were censored during the statistical analysis of HCC recurrence. Cox proportional hazards regression model was used to assess predictors of recurrence-free survival. Hazard ratios (HR, crude and adjusted) and corresponding 95\% confidence intervals (CI) were calculated. Receiver operating characteristic (ROC) curve analysis was used to determine the optimal cutoff value of AFP. Statistics calculations were performed usingSPSS software version 17.0 (SPSS Inc., Chicago, IL, USA). A $\mathrm{p}<0.05$ was considered statistically significant.

\section{Results}

A total of 142 patients were analyzed. Patients' demographic characteristics and histopathology results of explanted liver are shown in Tables 1 and 2, respectively. The median follow-up after LDLT was 41.9 months (IQR: 41.0 months). HCC recurred in 13 patients $(9.2 \%)$ at a median of 22.5 months after LDLT (IQR: 41.8 months). Ten recurrences occurred within 3 years, and 3 recurrences occurred after more than 5 years. The cumulative 1-, 3-, and 5-year OS were $96.4 \%, 90.1 \%$, and $84.0 \%$, respectively. The cumulative 1-, 3-, and 5-year RFS were $96.4 \%, 91.8 \%$, and $91.8 \%$, respectively.

Gender, MELD score, pre-transplant therapy, pre-transplant serum AFP level (including Log10(AFP), AFP $>20 \mathrm{ng} / \mathrm{ml}$, AFP $>100 \mathrm{ng} / \mathrm{ml}$, AFP $>200 \mathrm{ng} / \mathrm{ml}$ ), tumor number, and total tumor size $>10 \mathrm{~cm}$ were all significant predictors for a worse RFS (Table 3). When significant predictors were applied to the multivariable analysis, MELD score (adjusted HR: 1.16, 95\% CI: 1.04-1.25, $\mathrm{p}=0.006$ ) and pre-transplant Log10 (AFP) (adjusted HR: 3.14, 95\% CI: $1.46-6.76, \mathrm{p}=0.003$ ) were significant independent predictors associated with a worse recurrence-free survival (Table 3 ).

In the ROC curve analysis, pre-transplant serum AFP level is a significant predictor with an AUC of 0.76 (95\% CI $0.62-0.91 \mathrm{p}=0.002$ ) and the optimal cutoff values of AFP is $26 \mathrm{ng} / \mathrm{ml}$ with sensitivity of $76.9 \%$ and specificity of $71.3 \%$ (Figure 1). 
Table 2. Histopathology results of explanted liver.

\begin{tabular}{lc}
\hline Variables & Patients $(\mathbf{n}=\mathbf{1 4 2})$ \\
\hline Number of tumors & \\
Median (IQR) & $2(2)$ \\
$\leq 3$ & $113(79.6 \%)$ \\
$>3$ & $29(20.4 \%)$ \\
Largest tumor size (cm) & \\
Median (IQR) & $2.5(1.5)$ \\
$\leq 5 \mathrm{~cm}$ & $136(95.8 \%)$ \\
$>5 \mathrm{~cm}$ & $6(4.2 \%)$ \\
Total tumor size (cm) & \\
Median (IQR) & $4.0(4.3)$ \\
$\leq 10 \mathrm{~cm}$ & $130(91.5 \%)$ \\
$>10 \mathrm{~cm}$ & $12(8.5 \%)$ \\
Tumor differentiation & $14(9.9 \%)$ \\
Well differentiated & $101(71.1 \%)$ \\
Moderately differentiated & 0 \\
Poorly differentiated & $27(19.0 \%)$ \\
Uncertain* & \\
mVI & $95(66.9 \%)$ \\
Absent & $47(33.1 \%)$ \\
Present & \\
Milan criteria & $92(64.8 \%)$ \\
Within & $50(35.2 \%)$ \\
Beyond & \\
UCSF criteria & $108(76.1 \%)$ \\
Within & $34(23.9 \%)$ \\
\hline Beyond & \\
\hline & \\
\hline &
\end{tabular}

IQR, interquartile range; mVI, microvascular invasion; UCSF, University of California San Francisco. ${ }^{\star}$ Uncertain: undetermined because of extensive necrosis.

\section{Discussion}

Liver transplantation is the only treatment capable of treating both liver cancer and the underlying cirrhosis. But with the stable number of donors and constantly growing number of patients needing the liver transplant there is an evident need for formulating a system that allows to identify patients who will benefit most from the LT. Ideally, HCC patients undergoing LT should have outcomes comparable to patients transplanted for other indications. Unfortunately, this objective is still difficult to achieve [8]. The idea of performing a LT to treat cancer is based on an assumption that performing a complicated surgical procedure that uses a scarcely available organ will result in a significant improvement in patients' outcome. In the setting of LDLT the ethical consideration of exposing a health donor to a significant risk is also of utmost importance. Therefore, it seems logical that the criteria used for the selection of patients allows to identify those that have a possibility of survival similar to the patients transplanted for non-HCC indications [9].

The most commonly accepted criteria for including patients with HCC for transplantation are the Milan and

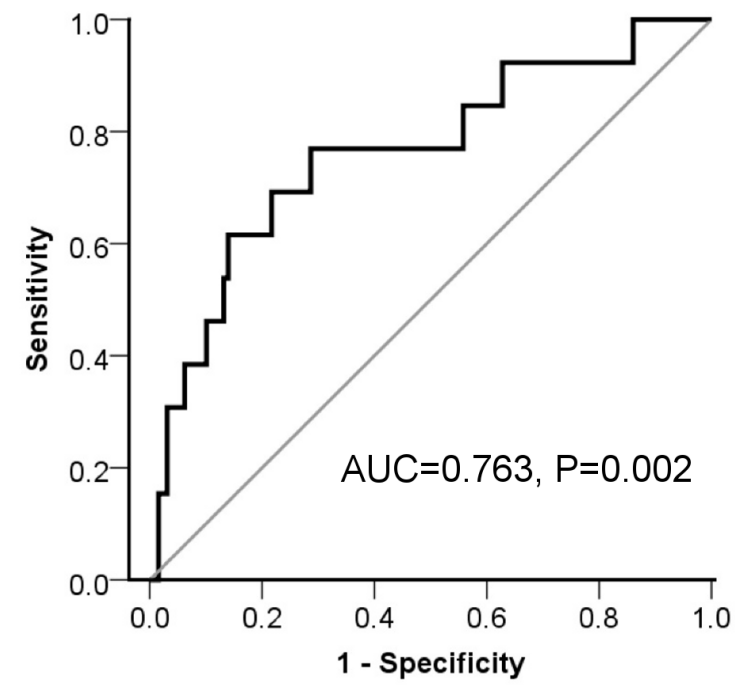

Figure 1. The receiver operating characteristic curve analyses for predicting HCC recurrence. Area under the curve of AFP was 0.763 and the optimal cutoff value was $26 \mathrm{ng} / \mathrm{ml}$ with sensitivity of $76.9 \%$ and specificity of $71.3 \%$.

UCSF criteria. For staging purposes, the AJCC/TNM system is also used. All staging systems for HCC are based on the tumor burden. In the AJCC/TNM system the stage is based on the size, number and infiltration of adjacent organs. In the current version this classification suggests recording the AFP level but its result has no effect on the final staging [10]. The Milan criteria and UCSF criteria do not mention AFP level at all $[2,3]$. This is also the case for current guidelines on HCC treatment [11]. On the other hand, in a recent report analyzing over 45,000 liver transplant patients performed in the United States a strong dose response correlation between the serum AFP level and post-transplant mortality has been shown [4]. The poorer survival in patients with HCC compared to patients transplanted for non-HCC indications was noticed already at AFP levels as low as $15 \mathrm{ng} / \mathrm{mL}$. The combination of total tumor volume and AFP level was also found to correlate with OS in transplanted HCC patients [12]. These findings suggest that including pre-transplantation AFP level in the staging systems could help identify patients at lower risk of developing HCC recurrence or dying from the disease who therefore would be better candidates for LT.

Patients with $\mathrm{HCV}$ infection tend to present with advanced cirrhosis and experience lower 5 -year survival than patients with non-HCV related disease [5]. Patients transplanted for $\mathrm{HCV}$ cirrhosis were initially thought to have worse outcomes after LDLT than after DLT [13]. However, more recent data does not support this statement confirming similar results for LDLT and deceased donor liver transplantation (DDLT) [14]. But still, the presence of HCV infection has been found to be the only factor influencing overall survival of patients undergoing LDLT with a HR of 2.29 [15]. 
Table 3. Uni- and multi-variable analyses (Cox proportional hazard model) for tumor recurrence after LDLT.

\begin{tabular}{|c|c|c|c|c|c|c|}
\hline \multirow{2}{*}{ Variables } & \multicolumn{3}{|c|}{ Univariable } & \multicolumn{3}{|c|}{ Multivariable } \\
\hline & HR & $95 \% \mathrm{CI}$ & p-value & HR & $95 \% \mathrm{CI}$ & p-value \\
\hline Age $>57$ y & 1.73 & $0.55-5.49$ & 352 & & & \\
\hline Gender (man) & 7.89 & $1.02-61.09$ & 48 & 8.00 & $0.96-66.68$ & 55 \\
\hline Diabetes & 0.65 & $0.14-2.99$ & 575 & & & \\
\hline MELD score & 1.09 & $1.01-1.17$ & 19 & 1.16 & $1.04-1.28$ & 6 \\
\hline Combine $\mathrm{HBV}$ infection & 1.20 & $0.33-4.40$ & 781 & & & \\
\hline Pre-transplant therapy & 0.28 & $0.09-0.86$ & 26 & 0.34 & $0.08-1.37$ & 129 \\
\hline Pre-transplant AFP & & & & & & 3 \\
\hline $\log 10($ AFP $)$ & 2.72 & $1.47-5.04$ & 0.001 & 3.14 & $1.46-6.76$ & \\
\hline $\mathrm{AFP}>20 \mathrm{ng} / \mathrm{ml}$ & 5.08 & $1.39-18.59$ & 0.014 & & & \\
\hline $\mathrm{AFP}>100 \mathrm{ng} / \mathrm{ml}$ & 4.01 & $1.28-12.53$ & 0.017 & & & \\
\hline $\mathrm{AFP}>200 \mathrm{ng} / \mathrm{ml}$ & 4.43 & $1.29-15.22$ & 0.018 & & & \\
\hline Tumor number & 1.34 & $1.13-1.60$ & 0.001 & 1.52 & $0.93-2.48$ & 94 \\
\hline Number $>3$ & 3.00 & $0.94-9.50$ & 0.063 & & & \\
\hline Largest tumor size $(\mathrm{cm})$ & 1.19 & $0.82-1.74$ & 0.364 & & & \\
\hline Largest size $>5 \mathrm{~cm}$ & 1.66 & $0.21-13.10$ & 0.629 & & & \\
\hline Total tumor size $(\mathrm{cm})$ & 1.23 & $1.09-1.40$ & 0.001 & & & 374 \\
\hline Total size $>10 \mathrm{~cm}$ & 8.45 & $2.67-26.73$ & $<0.001$ & 0.24 & $0.01-5.59$ & \\
\hline Tumor grade (MD) & 4.80 & $0.62-37.00$ & 132 & & & \\
\hline $\mathrm{mVI}$ & 1.88 & $0.63-5.60$ & 260 & & & \\
\hline Beyond Milan & 1.65 & $0.55-4.92$ & 373 & & & \\
\hline Beyond UCSF & 2.14 & $0.70-6.59$ & 185 & & & \\
\hline
\end{tabular}

MELD, Model for End-Stage Liver Disease; HBV, hepatitis B virus; AFP, serum $\alpha$-fetoprotein; MD, moderately differentiated ; mVI, microvascular invasion; UCSF, University of California San Francisco.

In this study, our aim was to evaluate whether preoperative AFP levels are better predictors of survival than tumor burden. The influence of donor-related factors was minimized in our study group. All patients were of Asian origin, all had underlying $\mathrm{HCV}$ infection and confirmed HCC (fulfilling in $76 \%$ of patients UCSF criteria). All patients were treated by LDLT performed by the same team and using the same technique. The influence of donor factors and intraoperative factors like cold isquemia time, warm isquemia time and surgical technique was therefore minimized. This is important since in a recent study longer cold ischemia time and warm ischemia time were both correlated to poorer survival of patients with HCC undergoing DDLT [16]. Among many factors influencing outcome of LT, the donor variables play an important role. The recipients of a liver graft from cardiac death donors had a higher probability of liver abscess, biliary stricture, and death 3 and 5 years after transplantation for $\mathrm{HCV}$ related liver disease compared with patients receiving grafts form brain dead donors. Shorter survival has been also noted for patients receiving grafts from deceased donors of more than 60 years of age [17]. All above mentioned technical and donor factors were eliminated or at least minimized in our series due to its high homogeneity.

In the presented group, $74 \%$ of patients received a downstaging treatment (RFA, TACE, PEI or surgery) prior to surgery. Pre-transplant therapy was found to influence recurrence after LDLT only in univariable analysis. This finding coincides with data from literature where the results of LDLT in patients who initially did not meet Milan or UCSF criteria but were downstaged before the transplant are equal to those fulfilling the criteria and not requiring any bridge therapy before transplantation $[3,18]$. The inability of downstaging a tumor into Milan criteria before LT has been shown in multivariable analysis to be an independent predictor of HCC recurrence [19]. This phenomenon was not noted in our series.

Our study confirmed a relationship between the pre-transplant AFP level and tumor recurrence with the optimal cut-off value of $26 \mathrm{ng} / \mathrm{mL}$. The correlation between pre-transplant AFP level and OS and RFS in patients undergoing LT for HCC has been shown in several studies. In a recent paper analyzing data of 45,000 patients transplanted for HCC, the impact on survival has been seen already at AFP levels of $16 \mathrm{ng} / \mathrm{mL}$ [4]. Also in smaller groups, this effect of AFP has been noted, although at higher concentrations of $400 \mathrm{ng} / \mathrm{mL}$ [12]. Maximum AFP has been noted to increase HR for tumor recurrence at a pace of 1.21 per log unit [19]. In our series, the adjusted HR for pre-transplant Log10 AFP was 3.14. Based on the analysis on 211 patients transplanted for HCC, Hameed et al. suggested that the cutoff value of AFP in candidates for liver transplantation fulfilling the Milan criteria should be as high as $1000 \mathrm{ng} / \mathrm{ml}$ [20]. In our study group on the other hand, the optimal cut-off value for AFP was $26 \mathrm{ng} / \mathrm{mL}$. The interpretation of these differences is not clear since in the paper by Hameed et al. only $62 \%$ of 
patients had HCV infection and in our group only $65 \%$ of patients fulfilled the Milan criteria. Also, the median AFP level in our patients was 14.2 and 10 patients (7\%) had an AFP level $>200$, while in Hameed's group median was 11 and $4.7 \%$ of patients had an AFP of $>1000$. Apart from AFP other biomarkers can be used for HCC surveillance. The evaluation of AFP-L3, DCP and the combination of its results with AFP level have been shown to yield promising results [21].

The tumor burden expressed by number of tumors and overall tumor size were found to influence RFS only in univariable analysis. The high tumor burden in the presented group is reflected by $76 \%$ of patients fulfilling UCSF criteria and only $65 \%$ of patients fulfilling the Milan criteria. No difference in outcome between those patients and patients who were outside the Milan, but within UCSF criteria, was noted. This finding is in contrast to the finding by Agopian et al. who found that non-downstaging HCC patient to fit Milan criteria before LT is a predictor of recurrence in multivariable analysis [19]. Median time to recurrence was longer in our group (15 months vs. 22 months) as well as OS and RFS at 1, 3 and 5 years. Although our study groups are not entirely comparable, this finding can be used as another argument against the use of Milan criteria. We have shown that patients undergoing LDLT who were beyond the Milan criteria even after bridge therapies were doing similar to the patients fulfilling those criteria. Based on these findings, it seems reasonable to state that UCSF criteria better define the group which is likely to survive for a longer time after transplantation.

Micro and macrovascular invasion has been shown in several reports to be a predictor of recurrence of HCC after LT $[19,20,22]$. In the present series, we have not found microvascular invasion to influence recurrence in univariable analysis. The level of AFP has been found to predict microvascular invasion in one study [20]. We have failed to confirm this correlation in our study group.

We have found the MELD score to be an independent factor of tumor recurrence in both, univariable and multivariable analyses. This confirms the findings from the recent study where MELD score together with HCV status, age of both donor and recipient and AFP level were found to influence graft and patient survival after DDLT [16].

There are several weaknesses in our study. One fourth of our study population did not meet UCSF criteria for liver transplantation, while $65 \%$ stayed within the stricter Milan criteria. This group represents therefore a wide spectrum of tumor burden. All other patient, surgeon and hospital-related factors are homogenous compared to other reported series. This high homogeneity (with the exclusion of tumor burden) of the study population can be seen as an advantage, but also makes it difficult to extrapolate our results to other patient groups. There were several techniques of downstaging used (e.g.: TACE, RFA, PEI, resection) which in many patients resulted in important fall in AFP level measured preoperatively. Both, the OS and RFS can vary depending on the technique of downstaging used [19]. In one systematic review comparing TACE with TARE (transarterial radio embolization - technique that had not been used in our study population), the results of both techniques were similar with $40 \%$ of success rate and 16\% recurrent HCC [23]. However, due to small numbers of patients in each of the pre-transplant therapies we could only compare all downstaging techniques to no downstaging.

Our findings strongly support the use of the pre-transplant AFP level as a determinant of post-transplantation outcome in patients with HCC and HCV related disease. The optimal cut-off value for specific group of patients has yet to be defined. In this series, the AFP and MELD score have been shown to influence HCC recurrence, while tumor burden was found to be important only in univariable analysis.

\section{Conclusion}

The pre-transplant AFP level and MELD score influence RFS in patients with HCC and HCV-related liver disease undergoing LDLT.

Acknowledgements: ALK, KDJ and BV where Liver Surgery and Transplantation Fellows at the Liver Transplantation Program and Department of Surgery, Kaohsiung Chang Gung Memorial Hospital, Kaohsiung, Taiwan during the preparation of this manuscript.

\section{References}

[1] EL-SERAG HB. Hepatocellular carcinoma. N Engl J Med. 2011; 365: 1118-1127. https://doi.org/10.1056/NEJMra1001683

[2] MAZZAFERRO V, REGALIA E, DOCI R, ANDREOLA S, PULVIRENTI A et al. Liver transplantation for the treatment of small hepatocellular carcinomas in patients with cirrhosis. N Engl J Med 1996; 334: 693-699. https://doi.org/10.1056/ NEJM199603143341104

[3] YAO FY, XIAO L, BASS NM, KERLAN R, ASCHER NL et al. Liver transplantation for hepatocellular carcinoma: validation of the UCSF-expanded criteria based on preoperative imaging. Am J Transplant 2007; 7: 2587-2596. https://doi. org/10.1111/j.1600-6143.2007.01965.x

[4] BERRY K, IOANNOU GN. Serum alpha-fetoprotein level independently predicts posttransplant survival in patients with hepatocellular carcinoma. Liver Transpl 2013; 19: 634645. https://doi.org/10.1002/lt.23652

[5] BARAZANI Y, HIATT JR, TONG MJ, BUSUTTIL RW. Chronic viral hepatitis and hepatocellular carcinoma. World J Surg 2007; 31: 1243-1248. https://doi.org/10.1007/s00268007-9041-3

[6] CHEN CL, CHEN YS, DE VILLA VH, WANG CC, LIN CL et al. Minimal blood loss living donor hepatectomy. Transplantation 2000; 69: 2580-2586.

[7] LIN TS, CONCEJERO AM, CHEN CL, CHIANG YC, WANG CC et al. Routine microsurgical biliary reconstruction decreases early anastomotic complications in living donor liver transplantation. Liver Transpl 2009; 15: 1766-1775. https://doi.org/10.1002/lt.21947 
[8] MAILEY B, BUCHBERG B, PRENDERGAST C, ARTINYAN A, KHALILI J et al. A disease-based comparison of liver transplantation outcomes. Am Surg 2009; 75: 901-908.

[9] POMFRET EA, WASHBURN K, WALD C, NALESNIK MA, DOUGLAS D et al. Report of a national conference on liver allocation in patients with hepatocellular carcinoma in the United States. Liver Transpl 2010; 16: 262-278. https:// doi.org/10.1002/lt.21999

[10] EDGE SB, BYRD DR, COMPTON CC, FRITZ AG, GREENE FL, TROTTI A (Eds.), AJCC Cancer Staging Handbook From the AJCC Cancer Staging Manual, 7th edition. Springer New York Dordrecht Heidelberg London, 2010, p. 648. ISBN: 9781845974749.

[11] EUROPEAN ASSOCIATION FOR THE STUDY OF THE LIVER; EUROPEAN ORGANISATION FOR RESEARCH AND TREATMENT OF CANCER. EASL-EORTC clinical practice guidelines: management of hepatocellular carcinoma. J Hepatol 2012; 56: 908-943. https://doi.org/10.1016/j. jhep.2011.12.001

[12] GRAT M, KORNASIEWICZ O, HOLOWKO W, LEWANDOWSKI Z, ZIENIEWICZ K et al. Evaluation of total tumor volume and pretransplantation $\alpha$-fetoprotein level as selection criteria for liver transplantation in patients with hepatocellular cancer. Transplant Proc 2013; 45: 1899-1903. https:// doi.org/10.1016/j.transproceed.2012.12.010

[13] GARCIA-RETORTILLO M, FORNS X, LLOVET JM, NAVASA M, FELIU A et al. Hepatitis C recurrence is more severe after living donor compared to cadaveric liver transplantation. Hepatology 2004; 40: 699-707. https://doi.org/10.1002/ hep. 20357

[14] TERRAULT NA, SHIFFMAN ML, LOK AS, SAAB S, TONG L et al. Outcomes in hepatitis $C$ virus-infected recipients of living donor vs. deceased donor liver transplantation. Liver Transpl 2007; 13: 122-129. https://doi.org/10.1002/ lt.20995

[15] REICHMAN TW, KATCHMAN H, TANAKA T, GREIG PD, MCGILVRAY ID et al. Living donor versus deceased donor liver transplantation: a surgeon-matched comparison of recipient morbidity and outcomes. Transpl Int 2013; 26: 780-787. https://doi.org/10.1111/tri.12127
[16] CROOME KP, WALL W, CHANDOK N, BECK G, MAROTTA $P$ et al. Inferior survival in liver transplant recipients with hepatocellular carcinoma receiving donation after cardiac death liver allografts. Liver Transpl 2013; 19: 12141223. https://doi.org/10.1002/lt.23715

[17] YAGCI G, FERNANDEZ LA, KNECHTLE SJ, D'ALESSANDRO AM, CHIN LT et al. The impact of donor variables on the outcome of orthotopic liver transplantation for hepatitis C. Transplant Proc 2008; 40: 219-223. https:// doi.org/10.1016/j.transproceed.2007.11.058

[18] LEI J, YAN L. Comparison between living donor liver transplantation recipients who met the Milan and UCSF criteria after successful downstaging therapies. J Gastrointest Surg 2012; 16: 2120-2125. https://doi.org/10.1007/s11605-0122019-y

[19] AGOPIAN VG, HARLANDER-LOCKE M, ZARRINPAR A, KALDAS FM, FARMER DG et al. A novel prognostic nomogram accurately predicts hepatocellular carcinoma recurrence after liver transplantation: analysis of 865 consecutive liver transplant recipients. J Am Coll Surg 2015; 220: 416-427. https://doi.org/10.1016/j.jamcollsurg.2014.12.025

[20] HAMEED B, MEHTA N, SAPISOCHIN G, ROBERTS JP, YAO FY. Alpha-fetoprotein level $>1000 \mathrm{ng} / \mathrm{mL}$ as an exclusion criterion for liver transplantation in patients with hepatocellular carcinoma meeting the Milan criteria. Liver Transpl 2014; 20: 945-951. https://doi.org/10.1002/lt.23904

[21] YOO T, LEE KW, YI NJ, CHOI YR, KIM H et al. Peri-Transplant Change in AFP Level: a Useful Predictor of Hepatocellular Carcinoma Recurrence Following Liver Transplantation. J Korean Med Sci 2016; 31: 1049-1054. https://doi. org/10.3346/jkms.2016.31.7.1049

[22] BEST J, BILGI H, HEIDER D, SCHOTTEN C, MANKA P et al. The GALAD scoring algorithm based on AFP, AFP-L3, and DCP significantly improves detection of BCLC early stage hepatocellular carcinoma. Z Gastroenterol 2016; 54: 1296-1305. https://doi.org/10.1055/s-0042-119529

[23] PARIKH ND, WALJEE AK, SINGAL AG. Downstaging hepatocellular carcinoma: A systematic review and pooled analysis. Liver Transpl 2015; 21: 1142-1152. https://doi. org/10.1002/lt.24169 